\title{
GROUP INVARIANT SOLUTIONS IN MATHEMATICAL PHYSICS AND DIFFERENTIAL GEOMETRY
}

\author{
I.M. Anderson, M. E. Fels, C.G. Torre
}

December 10, 2000

\section{INTRODUCTION.}

Inspired by Galois' theory of symmetry of polynomial equations, Sophus Lie (1842-1899) developed an analogous theory of symmetry for differential equations. Besides this leading to the mathematics of Lie groups and transformation groups, Lie's theory led to an algorithmic way to find special explicit solutions to differential equations with symmetry. These special solutions are called group invariant solutions and they constitute practically every known explicit solution to the systems of non-linear partial differential equations which arise in mathematical physics and differential geometry. For example the Schwarzschild solution to the vacuum Einstein equations, and the instanton solution to the (self-dual) Yang-Mills equations are both group invariant solutions.

Today the search for group invariant solutions is still a common approach to explicitly solving non-linear partial differential equations. For instance, interesting examples of Einstein manifolds, harmonic maps and Ricci-solitons with cohomogeniety one symmetry groups can be found in [4],[7] and [6].

An excellent introductory reference to Lie's theory of group invariant solutions is [8]. However in this treatment (as in others [3],[9]) the theory is developed to mainly study scalar partial differential equations and does not apply to the standard equations of mathematical physics. In fact one cannot even reproduce the Schwarzschild solution from the techniques provided by these references. The technical assumption made in these references which precludes these famous examples is known as transversality. In this article, which is more or less a summary of [1], we provide a general method to find group invariant solutions for non-transverse group actions. The method also resolves the question of "how many" differential equations determine the group invariant solutions, a question which does not arise for scalar equations.

1991 Mathematics Subject Classification. Primary 58J70,35A30; Secondary 35Q75.

Key words and phrases. Group Invariant Solutions, Kinematic Reduction Diagram, Dynamic Reduction Diagram, Transverse Group Actions.

Research supported in part by NSF Grants DMS-\#9804833 and PHY-\#0070867 


\section{Notation And Preliminaries.}

Let $\pi: E \rightarrow M$ be a fibre bundle with $n$-dimensional base, and $m$-dimensional fibre. The map $\pi$ in local coordinates has the form

$$
\pi\left(x^{i}, u^{\alpha}\right)=\left(x^{i}\right) \quad i=1 \ldots n, \alpha=1 \ldots m .
$$

The local coordinates $\left(x^{i}\right)$ on the base $M$, play the role of the independent variables, while the local coordinates $\left(u^{\alpha}\right)$ on the fibres will be the dependent variables. The sections of $E$ will be denoted by

$$
S(E)=\left\{s: M \rightarrow E, \quad \pi \circ s=I_{M}\right\}
$$

and $s \in S(E)$ takes the form $u^{\alpha}=u^{\alpha}(x)$ in local coordinates.

A group $G$ acting on $E$ is said to act projectably (or by fibre preserving transformations) if there exists an action of $G$ on $M$ such that for all $g \in G$ the diagram

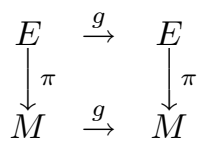

commutes. The notation $g p$ and $g x$ denotes the action of $G$ on $E$ and $M$ respectively.

Given a group $G$ acting projectably on $E$ ( and hence acting on $M$ ) and a point $x \in M$ then the isotropy or stabilizer subgroup of $x$ is

$$
G_{x}=\{g \in G, \mid g x=x\} .
$$

For each $x \in M$ the subgroup $G_{x}$ acts on the fibre $E_{x}$. That is given $x \in M$ and $g \in G_{x}$, and $p \in E_{x}=\pi^{-1}(x)$ then as a consequence of the commutative diagram (1) we find

$$
\pi(g p)=g \pi(p)=g x=x=\pi(p)
$$

and hence $g p \in E_{x}$.

\section{Examples Part I.}

Example 1.a. Let $G=S O(3)$ act on $M=N=\mathbb{R}^{3}-0$ in the usual way, and let $E$ be the trivial bundle $\pi: M \times N \rightarrow M$. The action of $G$ on $E$ is

$$
R(\mathbf{x}, \mathbf{u})=(R \mathbf{x}, R \mathbf{u}) \quad \mathbf{x} \in M, \mathbf{u} \in N, R \in S O(3) .
$$

This action is projectable with the projected action of $G$ on $M$ being the one given.

Example 1.b. Let $G$ be any Lie group acting on manifolds $M$ and $N$. Let $E$ be the trivial bundle $\pi: M \times N \rightarrow M$. Take the action of $G$ on $E$ to be the product action

$$
g(\mathbf{x}, \mathbf{u})=(g \mathbf{x}, g \mathbf{u}) \quad \mathbf{x} \in M, \mathbf{u} \in N, g \in G
$$

which is projectable.

Example 2.a. Let $M$ be any differentiable manifold and let $G=\operatorname{diff}(M)$ be the group of diffeomorphisms of $M$, and let $E$ be any tensor-bundle over $M$. The action of $\operatorname{diff}(M)$ on $M$ lifts to a natural action of $\operatorname{diff}(M)$ on $E$, which in the case $E=T M$ and $\phi \in \operatorname{diff}(M)$ is $\phi_{*}: T M \rightarrow T M$. These lifted actions are projectable.

Example 2.b. Let $G \subset \operatorname{diff}(M)$ be any Lie group, then the lift of this action to any tensor-bundle over $M$ as in example 2.a is projectable. 


\section{INVARIANT SECTIONS.}

Given a group $G$ acting projectably on $E$ there is an induced action of $G$ on $S(E)$ the sections of $E$. Given $s \in S(E)$ and $g \in G$ then the section $g \cdot s$ is defined by

$$
(g \cdot s)(x)=g s\left(g^{-1} x\right), \quad \forall x \in M .
$$

Definition 1. Let $G$ act projectably on $E$. A section $s \in S(E)$ is $G$-invariant if $g \cdot s=s$ or

$$
g s(x)=s(g x) .
$$

Denote by $S(E)^{G}$ the set of $G$-invariant sections of $E$, where of course $S(E)^{G}$ is the set of fixed points of the action of $G$ on $S(E)$.

Example 1.b. (continued) The group $G$ acts on each term separately in the product bundle $E=M \times N \rightarrow M$. In this case the space $S(E)^{G}$ admits an alternate description. Given $\phi \in C^{\infty}(M, N)$, the smooth maps from $M$ to $N$, we define a section $s_{\phi}: M \rightarrow E$ by $s_{\phi}(x)=(x, \phi(x))$, which identifies

$$
S(E)=C^{\infty}(M, N)
$$

Using this identification, a section $s \in S(E)$ is invariant if and only if

$$
g s_{\phi}(x)=(g x, g \phi(x))=(g x, \phi(g x))=s_{\phi}(g x)
$$

or $\phi(g x)=g \phi(x)$. Thus the invariant sections of $E$ are just the $G$-equivariant maps $\phi: M \rightarrow N$ and for this reason group invariant solutions are sometimes called equivariant [7].

A fundamental problem in the theory of group invariant solutions is how to parameterize the space $S(E)^{G}$. Adding the following hypothesis, which is not often valid but whose definition will be used later, makes this fairly easy.

Definition 2. A projectable group action $G$ on $E$, is said to be transverse if for all $p \in E$

$$
\pi(g p)=\pi(p) \quad \text { implies } \quad g p=p .
$$

Equivalently, $G$ acts transversely on $E$ if the orbits of $G$ in $E$ project diffeomorphically under $\pi$ to the orbits of $G$ in $M$. In local coordinates if the infinitesimal generators of the action of $G$ on $E$ are given by

$$
\Gamma=\left\{\xi_{a}^{i}(\mathbf{x}) \partial_{x^{i}}+\phi_{a}^{\alpha}(\mathbf{x}, \mathbf{u}) \partial_{u^{\alpha}}\right\} \quad a=1 \ldots \operatorname{dim} G
$$

and if the action is transverse then

$$
\operatorname{rank}\left[\xi_{a}^{i}(\mathbf{x})\right]=\operatorname{rank}\left[\xi_{a}^{i}(\mathbf{x}), \phi_{a}^{\alpha}(\mathbf{x}, \mathbf{u})\right]
$$

The invariant sections $S(E)^{G}$ for transverse actions are easily parameterized. 
Theorem 1. Let $G$ be a Lie group acting projectably and transversally on the bundle $\pi: E \rightarrow M$, and regularly on $M$ (so that $M / G$ is a manifold). Then $S(E)^{G}$ is in one-to-one correspondence with the sections of the bundle $E / G \rightarrow M / G$

Proof. We will only show how a section $\tilde{s}: M / G \rightarrow E / G$ defines a section $s: M \rightarrow$ $E$, and leave the rest as an exercise. Given $\tilde{x} \in M / G$ let $x \in M$ with $\mathfrak{q}_{M}(x)=\tilde{x}$ where $\mathfrak{q}_{M}: M \rightarrow M / G$. First we claim there exists a unique $p \in E$ such that

$$
\left.i] \mathfrak{q}_{E}(p)=\tilde{s}(\tilde{x}) \quad i i\right] \pi(p)=x
$$

where $\mathfrak{q}_{E}: E \rightarrow E / G$. Suppose there exist $p, p^{\prime}$ satisfying conditions $\left.\left.i\right], i i\right]$. By condition $i] p^{\prime}=g p$ for some $g \in G$, which when used in condition $\left.i i\right]$ along with transversality (3) implies $p^{\prime}=p$. Therefore define $s(x)=p$, to get the appropriate section. See [2] for more details.

The following simple corollary demonstrates that transverse group actions are unusual, and hence most examples of group actions on bundles are not transverse. the following.

Corollary 1. Let $G$ act projectably on $E$, then $G$ acts transversally if and only if for all $x \in M, G_{x}$ acts trivially on $E_{x}$.

For the examples we are interested in we need to find a way to parameterize the space $S(E)^{G}$ when the action of $G$ on $E$ may not be transverse. In order to do so, we use the following observation. Let $s \in S(E)^{G}$ be an invariant section, let $x \in M$ and let $g \in G_{x}$. The invariant section condition (2) at $x$ gives

$$
g s(x)=s(g x)=s(x)
$$

with the last identity coming from $g \in G_{x}$. Therefore if $s \in S(E)^{G}$ then $s(x) \in$ $\left(E_{x}\right)^{G_{x}}$, the fixed point set of $G_{x}$ acting on $E_{x}$, which motivates the following definition.

Definition 3. Let $G$ act projectably on $E$ and let $\kappa_{x}(E)=\left(E_{x}\right)^{G_{x}}$ and

$$
\kappa(E)=\bigcup_{x \in M} \kappa_{x}(E) .
$$

We call $\pi: \kappa(E) \rightarrow M$ the kinematic bundle. ${ }^{1}$

A simple consequence of this definition and equation (4) is

Corollary 2. Every $s \in S(E)^{G}$ factors through $\pi: \kappa(E) \rightarrow M$.

Note that this corollary does not say that every section of $\kappa(E) \rightarrow M$ is an element of $S(E)^{G}$. However the following is true,

Theorem 2. The subset $\kappa(E) \subset E$ is $G$-invariant, $G$ acts transversally on $\pi$ : $\kappa(E) \rightarrow M$ and $S(E)^{G}=S(\kappa(E))^{G}$.

Proof. Let $p \in \kappa(E)$ with $x=\pi(p)$ and let $g \in G, h \in G_{g x}$. The subgroup $G_{g x}=g G_{x} g^{-1}$ therefore $h=g h^{\prime} g^{-1}$ for some $h^{\prime} \in G_{x}$ and

$$
h g p=g h^{\prime} g^{-1} g p=g h^{\prime} p=g p
$$

so $g p \in \kappa_{g x}(E)$. Transversality follows directly from the definition of $\kappa(E)$.

Theorem 2 along with Theorem 1 imply the next theorem.

\footnotetext{
${ }^{1}$ The set $\kappa(E)$ is not necessarily a manifold, but for our discussion we will assume it is and that $\pi: \kappa(E) \rightarrow M$ is a submersion.
} 
Theorem 3. Suppose $\kappa(E) \rightarrow M$ is a bundle, and that $M / G$ is a manifold, then the space $S(E)^{G}$ of smooth invariant sections is in one-to-one correspondence with smooth sections of $\kappa(E) / G \rightarrow M / G$.

The situation is neatly summarized by the diagram,

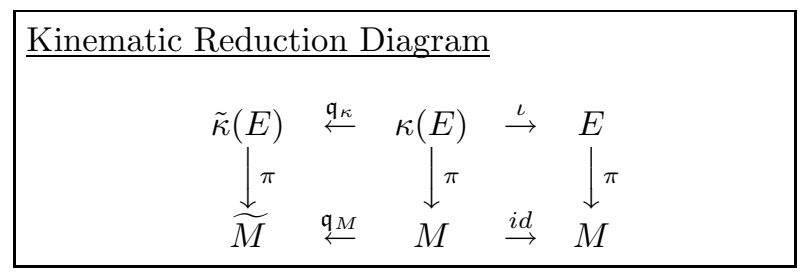

where $\tilde{\kappa}(E)=\kappa(E) / G$ and $\widetilde{M}=M / G$, and every section of $\tilde{\kappa}(E) \rightarrow \widetilde{M}$ lifts to an invariant section of $E \rightarrow M$.

It is worth emphasizing that the invariant sections of a bundle with a nontransverse group action are parameterized by the bundle $\tilde{\kappa}(E) \rightarrow \widetilde{M}$ whose base and fibre are typically of smaller dimension than $E \rightarrow M$.

\section{Examples Part II - Kinematic Reduction.}

Example 1.a. (continued) $G=S O(3)$, is acting on $M=N=\mathbb{R}^{3}-0$ in the standard way, and $E$ is the trivial bundle $\pi: M \times N \rightarrow M$. The isotropy $G_{\mathbf{x}}$ at $\mathbf{x} \in M$ is $S O(2)_{\mathbf{x}}$, the rotations about the line containing $\mathbf{x}$ and the origin. The set $\kappa_{\mathbf{x}}(E) \subset E$ is

$$
\kappa_{\mathbf{x}}(E)=\left\{(\mathbf{x}, \mathbf{u}) \mid R \mathbf{u}=\mathbf{u}, R \in S O(2)_{\mathbf{x}}\right\}
$$

and so $\mathbf{u} \propto \mathbf{x}$, or $\mathbf{u}=v \mathbf{x}, v \in \mathbb{R}$. The bundle $\kappa(E)$ is a trivial line bundle with coordinates $(\mathbf{x}, v)$ and the inclusion $\kappa(E) \rightarrow E$ given by $(\mathbf{x}, v) \rightarrow(\mathbf{x}, \mathbf{u}=v \mathbf{x})$. The function $r=\sqrt{x^{2}+y^{2}+z^{2}}$ defines a global coordinate on $M / G$. A coordinate description of the kinematic reduction diagram is

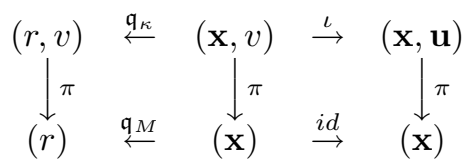

where $\iota(\mathbf{x}, v)=(\mathbf{x}, \mathbf{u}=v \mathbf{x})$. Any invariant section $v=v(r)$ of $\tilde{\kappa}(E) \rightarrow \widetilde{M}$ (the left hand side of (6)) lifts to the invariant section $u(\mathbf{x})=v(r) \mathbf{x}$ of $E$.

Example 2.b. (continued) Let $G=S O(3) \times\left(Z_{2} \ltimes \mathbb{R}\right)$ act on $\left(\mathbb{R}^{3}-0\right) \times \mathbb{R}$ with local coordinates $(x, y, z, t)$ by

$$
(R, \tau, \epsilon)(\mathbf{x}, t)=(R \mathbf{x}, \epsilon t+\tau) .
$$

The isotropy group at $\left(\mathbf{x}, t_{0}\right)$ is $G_{\left(\mathbf{x}, t_{0}\right)}=S O(2)_{\mathbf{x}} \times Z_{2}$ where the $Z_{2}$ action is

$$
(\mathbf{x}, t) \rightarrow\left(\mathbf{x}, \epsilon\left(t-t_{0}\right)+t_{0}\right) .
$$


Let $E=T^{*} M \odot T^{*} M$, the bundle of symmetric tensors over $M$. In this example the set $\kappa_{\left(\mathbf{x}, t_{0}\right)}(E)$ which we wish to compute, is just the set of invariants of the symmetric tensor product of the dual of the linear isotropy representation.

In order to compute $\kappa_{\left(\mathbf{x}, t_{0}\right)}(E)$ we consider each term in the product group $G_{\left(\mathbf{x}, t_{0}\right)}$ separately. For $R \in S O(2)_{\mathbf{x}}$, the condition for $\gamma \in \kappa_{\left(\mathbf{x}, t_{0}\right)}(E)$ is $R^{T} \gamma R=\gamma$. Since $S O(2)_{\mathrm{x}}$ is connected this condition can be written infinitesimally as

$$
X^{T} \gamma+\gamma X=0 \quad \text { where } \quad X=\left(\begin{array}{cccc}
0 & z & -y & 0 \\
-z & 0 & x & 0 \\
y & -x & 0 & 0 \\
0 & 0 & 0 & 0
\end{array}\right) \text {. }
$$

Solving these equations gives

$\gamma=A(x d x+y d y+z d z)^{2}+B\left(d x^{2}+d y^{2}+d z^{2}\right)+C d t^{2}+D(x d x+y d y+z d z) \odot d t$

where $A, B, C, D \in \mathbb{R}$. The constraint for the $Z_{2}$ part of the isotropy is $S^{T} \gamma S=\gamma$ where $S=\operatorname{diag}(0,0,0, \epsilon)$, which implies $D=0$. Therefore $\kappa_{\left(\mathbf{x}, t_{0}\right)}(E)=(A, B, C)$ and $\kappa(E)$ is a rank 3 vector subbundle of $E$. The kinematic reduction diagram is

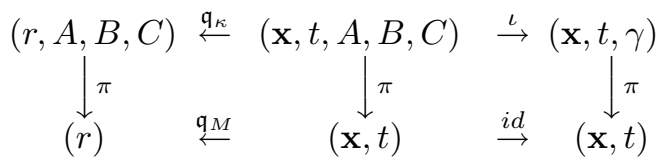

where $r=\sqrt{x^{2}+y^{2}+z^{2}}$, and $\iota(\mathbf{x}, t, A, B, C)=\left(\mathbf{x}, t, \gamma=A(r d r)^{2}+B\left(d x^{2}+d y^{2}+\right.\right.$ $\left.\left.d z^{2}\right)+C d t^{2}\right)$. Finally the sections $(A(r), B=B(r), C=C(r))$ of $\tilde{\kappa}(E)$ (the left side of diagram (7)) give rise to the $G$-invariant symmetric covariant 2 -tensors

$$
\gamma=A(r)(r d r)^{2}+B(r)\left(d x^{2}+d y^{2}+d z^{2}\right)+C(r) d t^{2} .
$$

\section{The Reduced Equations.}

Let $J^{k}(E)$ be the bundle of $k$-jets of sections of $E$ and let $G$ act projectably on $E$. Then $G$ acts on $J^{k}(E)$ by

$$
g \cdot \sigma=j^{k}(g \cdot s)(g x)
$$

where $\sigma=j^{k}(s)(x) \in J^{k}(E)$ and $j^{k}(s)$ is the $k^{t h}$-jet of a section $s \in S(E)$. A $k^{t h}$ order differential operator $\Delta$ is a section of a vector-bundle $\mathcal{D} \rightarrow J^{k}(E)$. A solution to the differential equations $\Delta=0$ is a section $s: M \rightarrow E$ such that $\Delta \circ j^{k}(s)=0$. Denote the solutions to $\Delta$ by the subset $S_{\Delta}(E) \subset S(E)$.

Let $G$ be a projectable group action on the bundle $\mathcal{D} \rightarrow J^{k}(E)$, then $G$ is a symmetry group of $\Delta$ if $\Delta: J^{k}(E) \rightarrow \mathcal{D}$ is a $G$-invariant section. It is easy to verify

Lemma 1. If $s \in S_{\Delta}(E)$ and $g \in G$ a symmetry group of $\Delta$, then $g \cdot s \in S_{\Delta}(E)$.

In other words if $G$ is a symmetry group of $\Delta$ then the subset $S_{\Delta}(E) \subset S(E)$ is $G$-invariant. The fixed point set $S_{\Delta}(E)^{G}$ of the action of $G$ on $S_{\Delta}(E)$ leads to the definition 
Definition 4. Let $G$ be a symmetry group for $\Delta$. A solution

$$
s \in S_{\Delta}(E) \bigcap S(E)^{G}=S_{\Delta}(E)^{G}
$$

is called a $G$-invariant solution.

We now determine a reduced differential operator $\widetilde{\Delta}$ whose solutions determine all the $G$-invariant solutions to $\Delta$. Every $G$-invariant solution to a differential equation is an element of $S(E)^{G}$ which by Theorem 3 can be identified with a section of $\tilde{\kappa}(E) \rightarrow \widetilde{M}$. Therefore the reduced differential operator will be a differential operator on $J^{k}(\tilde{\kappa}(E))$ and define a section of some vector-bundle $\widetilde{\mathcal{D}} \rightarrow J^{k}(\tilde{\kappa}(E))$.

It is not possible to directly apply the theory developed in section 4 to find the reduced differential operator and bundle $\widetilde{\mathcal{D}}$ for $\Delta$. We need to introduce the so-called invariant jet space $\operatorname{In} v^{k}(E)$. The set $\operatorname{Inv} v^{k}(E) \subset J^{k}(E)$ is defined by

$$
\operatorname{Inv}^{k}(E)=\left\{\sigma \in J^{k}(E) \text {, where } \sigma=j^{k}(s)(x) \text { for some } s \in S(E)^{G}\right\} .
$$

We list some facts about invariant jet-space spaces.

Lemma 2. Let $G$ act projectably on $E$ then

a] $\operatorname{Inv}^{k}(E) \subset J^{k}(E)$ is $G$-invariant.

b] $G$ acts transversally on $\operatorname{Inv}^{k}(E) \rightarrow M$.

c] Every $j^{k}(s): M \rightarrow J^{k}(E)$, for $s \in S(E)^{G}$ factors through $\operatorname{Inv}^{k}(E)$.

Lemma 3. If $\kappa(E)$ is a bundle and $M / G$ is a manifold then

a] $\operatorname{Inv}^{0}(E)$ is diffeomorphic to $\kappa(E)$.

b] $\operatorname{Inv}^{k}(E) \rightarrow M$ is equivalent to the pull-back bundle $\mathfrak{q}^{*} J^{k}(\tilde{\kappa}(E))$ where $\mathfrak{q}_{M}$ : $M \rightarrow M / G$.

Suppose that the condition in Lemma 3 hold, let $i: \operatorname{Inv}^{k}(E) \rightarrow J^{k}(E)$ be the inclusion map, and let $\mathcal{D}_{i} \rightarrow \operatorname{Inv} v^{k}(E)$ be the restriction (or pullback) of the vector-bundle $\mathcal{D} \rightarrow J^{k}(E)$ to $\operatorname{Inv}^{k}(E)$. Then we have,

Lemma 4. The group $G$ acts on the sub-bundle $i: \operatorname{Inv}^{k}(E) \rightarrow J^{k}(E)$ and $\Delta$ defines an invariant section of the restricted bundle $\mathcal{D}_{i} \rightarrow \operatorname{Inv}^{k}(E)$.

At this point we apply the theory of section 4 to the bundle $\mathcal{D}_{i} \rightarrow \operatorname{Inv} v^{k}(E)$ to obtain the left side in the diagram

$$
\begin{array}{|cccccccc|}
\hline \multicolumn{2}{|l}{\text { Dynamic Reduction Diagram }} \\
\kappa\left(\mathcal{D}_{i}\right) / G & \stackrel{\mathfrak{q}}{\leftarrow} & \kappa\left(\mathcal{D}_{i}\right) & \stackrel{\iota}{\rightarrow} & \mathcal{D}_{i} & \stackrel{i}{\rightarrow} & \mathcal{D} \\
\downarrow & & & \downarrow & & & \\
J^{k}(\tilde{\kappa}(E)) & \stackrel{\mathfrak{q}}{\leftarrow} & \operatorname{Inv}^{k}(E) & \stackrel{i d}{\rightarrow} & \operatorname{Inv}^{k}(E) & \stackrel{i}{\rightarrow} & E \\
\hline
\end{array}
$$

Theorem 3 states that the invariant sections of $\mathcal{D}_{i} \rightarrow \operatorname{Inv} v^{k}(E)$ are in one to one correspondence with sections of $\kappa\left(\mathcal{D}_{i}\right) / G \rightarrow J^{k}(\tilde{\kappa}(E))$. Given an invariant differential operator $\Delta$ we call the operator $\widetilde{\Delta}$ obtained through this correspondence, the reduced operator. 


\section{Examples Part III - Dynamic Reduction.}

Example 1.a. (continued) The Euler-Equations. Let $G=S O(3)$ act on $M=N=$ $\left(\mathbb{R}^{3}-0\right) \times \mathbb{R}$ in the standard way on the $\mathbb{R}^{3}-0$ term, and let $E$ be the trivial bundle $\pi: M \times N \rightarrow M$. We use $(\mathbf{x}, t)$ and $(\mathbf{u}, p)$ as coordinates on $M$ and $N$ respectively. The standard coordinates on $J^{1}(E)$ are $\left(\mathbf{x}, t, \mathbf{u}, p ; u^{i}{ }_{j}, u_{t}^{i}, p_{j}, p_{t}\right), i, j=1,2,3$.

Let $\mathcal{D}=\mathbb{R}^{4} \times J^{1}(E)$ be a trivial rank 4 vector bundle. We use $\left(\Delta^{i}, \Delta^{4}\right), i=$ $1,2,3$ for fibre coordinates, and take for the action of $G$ on the fibres $R\left(\Delta^{i}, \Delta^{4}\right)=$ $\left(R_{j}^{i} \Delta^{j}, \Delta^{4}\right), R \in S O(3)$. The Euler-equations are given by the vanishing of the differential operator $\Delta: J^{1}(E) \rightarrow \mathcal{D}$

$$
\begin{aligned}
\Delta^{i} & =u_{t}^{i}+u^{i}{ }_{j} u^{j}+\delta^{i j}\left(p,{ }_{j}\right) \quad i=1 \ldots 3 \\
\Delta^{4} & =u^{j}{ }_{j}
\end{aligned}
$$

where $\delta^{i j}$ are the components of the three dimensional (contravariant) Euclidean metric in standard coordinates.

To find the reduced operator we first determine $\operatorname{Inv}^{1}(E) \rightarrow J^{1}(E)$ using a slight extension of diagram (6). The invariant sections of $E$ are simply seen to be $S(E)^{G}=$ $\{\mathbf{u}=v(r, t) \mathbf{x}, p=p(r, t)\}$, where $r=\sqrt{x^{2}+y^{2}+z^{2}}$. The coordinates on $\operatorname{Inv}^{1}(E)$ are then $\left(\mathbf{x}, t, v, p, v_{r}, v_{t}, p_{r}, p_{t}\right)$ and the inclusion $i: \operatorname{Inv}^{1}(E) \rightarrow J^{1}(E)$ in coordinates is

$$
\begin{aligned}
& i:\left(\mathbf{x}, t, v, p, v_{r}, v_{t}, p_{r}, p_{t}\right) \rightarrow \\
& \left(\mathbf{x}, t, \mathbf{u}=v \mathbf{x}, p, u^{i},_{j}=r^{-1} v_{r} x^{i} x^{j}+v \delta_{j}^{i}, u_{t}^{i}=v_{t} x^{i}, p_{j}=r^{-1} p_{r} x^{j}, p_{t}=p_{t}\right) .
\end{aligned}
$$

To find the reduced operator we restrict $\Delta$ to $\operatorname{In} v^{1}(E)$ which gives

$$
\begin{aligned}
\Delta_{\text {Inv }}^{i} & =\left(v_{t}+v\left(v+r v_{r}\right)+r^{-1} p_{r}\right) x^{i} \\
\Delta_{\text {inv }}^{4} & =3 v+r v_{r} .
\end{aligned}
$$

From this $\kappa\left(\mathcal{D}_{i}\right)$ is a rank 2 bundle, and the components of the reduced operator are

$$
\tilde{\Delta}=\left(v_{t}+v\left(v+r v_{r}\right)+r^{-1} p_{r}, 3 v+r v_{r}\right) .
$$

Example 2.b. (continued) The Schwarzschild solution. Here $G=S O(3) \times Z_{2} \ltimes \mathbb{R}$, $M=\left(\mathbb{R}^{3}-0\right) \times \mathbb{R}$, and $E=T^{*} M \odot T^{*} M$. Using $\Pi: J^{2}(E) \rightarrow M$, let $\mathcal{D}=\Pi^{*} E$ be the pull-back bundle and let $G$ act on $\mathcal{D}$ in the natural way. In this example the differential operator $\Delta: J^{2}(E) \rightarrow \mathcal{D}$ is

$$
\Delta=R_{i j} d x^{i} \odot d x^{j}
$$

where $R_{i j}$ are functions of the second derivatives of a symmetric covariant two tensor, and $\Delta=0$ are the vacuum Einstein equations, or the Ricci-flat conditions.

The differential operator $\Delta$ takes values in $T^{*} M \odot T^{*} M$, so the bundle $\kappa\left(\mathcal{D}_{i}\right)$ will have the same structure as $\kappa(E)$ (see (7)). Thus restricting $\Delta$ to $\operatorname{Inv}^{2}(E)$ we find

$$
\Delta_{\text {Inv }}=\widetilde{\Delta}_{A}(x d x+y d y+z d z)^{2}+\widetilde{\Delta}_{B}\left(d x^{2}+d y^{2}+d z^{2}\right)+\widetilde{\Delta}_{C} d t^{2},
$$

where $\widetilde{\Delta}_{A}, \widetilde{\Delta}_{B}$, and $\widetilde{\Delta}_{C}$ are the components of the reduced operator. Solving the reduced equations $\widetilde{\Delta}_{A, B, C}=0$ leads to the Schwarzschild solution. 


\section{Quotients With BOUNDARY.}

In a number of applications, such as the cohomogeniety one reductions of harmonic map and Einstein equations [4],[7], the quotient $M / G$ is a manifold with boundary and consequently Theorem 3 does not hold and a general theory to parameterize the space $S(E)^{G}$ is unknown.

Example 4. Let $G=S O(2), M=N=S^{2} \subset \mathbb{R}^{3}, E=M \times N \rightarrow M$ where the action is by rotation about the $z$ axis on both $S^{2}$. The action is not transverse because at the poles $(0,0, \pm 1) \in M$ we have $G_{(0,0, \pm 1)}=S O(2)$ and this acts nontrivial on the $S^{2}$ fibre. The isotropy condition at $(0,0, \pm 1)$ leads to

$$
\kappa_{(0,0, \pm 1)}(E)=(0,0, \pm 1) .
$$

Therefore any $S O(2)$ equivariant map from $S^{2}$ to $S^{2}$ must take the poles to the poles. The quotient $M / G=[-1,1]$ and condition (9) would lead to constraints on the boundary conditions for any group invariant solution having this symmetry. See [1] for an explicit application to harmonic maps.

The above example shows that we can use the $\kappa$ "functor" to obtain information about the space of invariant sections of $E$ when $M / G$ is a manifold with boundary. Using the projection maps $\pi^{k}: J^{k}(E) \rightarrow M$ we can construct $\kappa\left(J^{k}(E)\right)$ where $\kappa_{x}\left(J^{k}(E)\right)=\left(J_{x}^{k}(E)\right)^{G_{x}}$. It is clear that $\operatorname{Inv}^{k}(E) \subset \kappa\left(J^{k}(E)\right)$ and so $\kappa\left(J^{k}(E)\right)$ constrains the $k$-jet of any invariant section. It is also possible to define $\kappa\left(J^{k}(E)\right)$ inductively using the projection maps $\pi_{k-1}^{k}: J^{k}(E) \rightarrow J^{k-1}(E)$. Let $\sigma \in \kappa\left(J^{k-1}(E)\right)$ then

$$
\kappa_{\sigma}\left(J^{k}(E)\right)=\left(\left(\pi_{k-1}^{k}\right)^{-1}(\sigma)\right)^{G_{\sigma}} .
$$

Note that $\kappa\left(J^{0}(E)\right)=\kappa(E)$.

Example 5. Let $G=S O(n), M=\mathbb{R}^{n}$, and $E=M \times \mathbb{R}$ so $S(E)^{G}$ is the space of $S O(n)$-invariant functions on $\mathbb{R}^{n}$, and $M / G=[0, \infty)$. Unlike the case when $M / G$ is manifold, the space $S(E)^{G}$ cannot be identified with the smooth functions $f: M / G \rightarrow \mathbb{R}$.

Let $\sigma \in J^{k-1}(E)$ with $\pi^{k-1}(\sigma)=\mathbf{0}$ the origin $\mathbb{R}^{n}$. We identify

$$
\left(\pi_{k-1}^{k}\right)^{-1}(\sigma)=\odot^{k}\left(\mathbb{R}^{n}\right)
$$

which represent the $k^{t h}$ order coefficients of the Taylor polynomial of a smoothfunctions $f$ defined in a neighbourhood of $\mathbf{0}$. We compute $\kappa_{\mathbf{0}}\left(J^{k}(E)\right)$ using (10). The stabilizer at $\mathbf{0}$ is $G_{\mathbf{0}}=S O(n)$ and for $\sigma \in \kappa\left(J^{k-1}(E)\right)$ with $\pi^{k-1}(\sigma)=\mathbf{0}$ we have

$$
\kappa_{\sigma}\left(J^{k}(E)\right)=\left(\odot^{k} \mathbb{R}^{n}\right)^{S O(n)},
$$

where the $S O(n)$ action is the $k^{\text {th }}$ order symmetric tensor product of the standard action of $S O(n)$ on $\mathbb{R}^{n}$. It is well known [11] that $\left(\odot^{k} \mathbb{R}^{n}\right)^{S O(n)}=0$ for $k$ odd. These necessary conditions are easily derived in even dimensions from the fact that if $f \in S(E)^{G}$ then $f(-\mathbf{x})=f(\mathbf{x})$, or $f(r)$ is even.

For examples where these conditions can be used to guarantee smoothness see [5] or [10]. 


\section{REFERENCES.}

[1] I.M. Anderson, M.E. Fels, C.G. Torre, Group invariant solutions without transversality, Comm. Math. Phys. 212 (2000), 653-686.

[2] I.M. Anderson, M.E. Fels, Transverse group actions on bundles, Submitted.

[3] G. W. Bluman and S. Kumei, Symmetries and differential equations, Applied Mathematical Sciences, 81, Springer-Verlag, New York-Derlin, 1989.

[4] A. Dancer, M. Wang, Integrable cases of the Einstein equations, Comm. Math. Phys. 208 (1999), 225-243.

[5] J. Eschenburg, M. Wang, The initial value problem for cohomegeniety one Einstein metrics, J. Geom. Analysis 10 (2000), 109-137.

[6] T. Ivey,, New examples of complete Ricci solitons, Proc. Amer. Math. Soc. 122(1) (1994), 241-245.

[7] H. Urakawa, Equivariant harmonic maps between compact Riemannian manifolds of cohomogenity 1, Michigan Math. J. 40 (1993), 27-50.

[8] P. J. Olver, Applications of Lie groups to differential equations, (Second Ed.), Springer, New York, 1986.

[9] H. Stephani, Differential equations and their solutions using symmetries (M. MacCallum, ed.), Cambridge University Press, Cambridge, 1989.

[10] M. Wang, Einstein metrics from symmetry and bundle constructions (to appear).

[11] H. Weyl, The classical groups their invariants and representations, Princeton University Press, Princeton, 1939.

Dept. of Mathematics and Statistics, Utah State University, Logan Utah, 84322

E-mail address: anderson@math.ams.org

Dept. of Mathematics and Statistics, Utah State University, Logan Utah, 84322

E-mail address: fels@math.ams.org

Dept. of Physics, Utah State University, Logan, Utah, 84322

E-mail address: torre@cc.usu.edu 